hep-th/9908106

CERN-TH-99-251

\title{
The Matrix model and the non-commutative geometry of the supermembrane
}

\author{
E.G. Floratos ${ }^{a, b, *}$ and G.K. Leontaris ${ }^{c, d, * *}$ \\ ${ }^{a}$ Institute of Nuclear Physics, NRCS Demokritos, Athens, Greece \\ ${ }^{b}$ Physics Department, University of Iraklion, Crete, Greece \\ ${ }^{c}$ Theoretical Physics Division, Ioannina University, GR-45110 Ioannina, Greece \\ ${ }^{d}$ CERN, Theory Division, 1211 Geneva 23, Switzerland
}

\begin{abstract}
This is a short note on the relation of the Matrix model with the non-commutative geometry of the 11-dimensional supermembrane. We put forward the idea that Mtheory is described by the 't Hooft topological expansion of the Matrix model in the large $N$-limit where all topologies of membranes appear. This expansion can faithfully be represented by the Moyal Yang-Mills theory of membranes. We discuss this conjecture in the case of finite $N$, where the non-commutative geometry of the membrane is given be the finite quantum mechanics. The use of the finite dimensional representations of the Heisenberg group reveals the cellular structure of a toroidal supermembrane on which the Matrix model appears as a non-commutatutive YangMills theory. The Moyal star product on the space of functions in the case of rational values of the Planck constant $\hbar$ represents exactly this cellular structure. We also discuss the integrability of the instanton sector as well as the topological charge and the corresponding Bogomol'nyi bound.
\end{abstract}

August 1999

*manolis@timaios.nuclear.demokritos.gr

**leonta@mail.cern.ch 


\section{Introduction}

One of the basic ingredients of M-threory [1, 2] is the eleven dimensional (11-d) supermembrane for which some years ago[3] a consistent action has been written in a general background of 11-d supergravity. The supermembrane has a uniquely defined self-interaction, which comes in contrast to the superstring, from a infinite dimensional gauge symmetry apparent in the light-cone gauge as the area-preserving diffeomorphisms on the surface of the membrane.

Because of the absence of the dilaton field for the supermembrane, there is no topological expansion over all possible three manifolds analogous to the string case. The supermembrane, due to its unique self-interaction, is possible to break into other supermembranes so in a sense is already a second quantized theory but up to now there is no consistent perturbative expansion. In the light-cone gauge, and flat space-time, there are two classes of membrane vacua, points and tensionless strings, so a low-energy effective field theory of supermembrane massless excitations would be either eleven-dimensional supergravity or a field theory for tensionless strings. Hopefully, recent efforts for understanding the coupling of 11-d supergravity with the supermembrane will help to the construction of its effective low energy field theory 4 .

In this letter, we present arguments that the Matrix model [5, 6, 7] describes the noncommutative geometry of the 11-d supermembrane, and M theory is the 't Hooft topological expansion of the Matrix model. We demostrate the existence of a topological charge and the corresponding Bogomol'nyi bound and we discuss the integrability of the instanton sector.

\section{Non-commutative geometry of the membrane}

It is a well known fact that the Matrix model[5, 6, 7] was one of the first ideas for the study of the dynamics of the bosonic membrane in the light-cone frame and in the approximation of finite number of oscillation modes [8, 9]. The true dynamics would be determined by taking the limit of infinite number of modes. In the finite mode approximation the Hamiltonian of the membrane is exactly the same with $S U(N)$ Yang-Mills (YM) classical mechanics and this system is known to possess interesting chaotic dynamics [10] and a discrete spectrum at the level of quantum mechanics (QM) [11]. Later on, Townsend et al [3, 12] discovered the supermembrane Lagrangian in 11 dimensions and the finite mode truncation, as was expected, is described by the Hamiltonian of the supersymmetric $S U(N)$ YM mechanics. It was found that the quantum mechanical spectrum of this model is continuous; at that time this was considered to be the end of the supermembrane as a fundamendal object replacing the superstring and producing all the low energy physics that could be useful for the unification of gauge and gravitational forces [13, 14].

In ref. [15] the question of a deeper origin of the $S U(N)$ YM classical mechanics as an approximation of the membrane dynamics was considered and it was found that $S U(N)$ rep-

resents the Lie algebra of the finite Heisenberg group, which acts on a discretized membrane 
representing a toroidal discrete phase space. The membrane coordinates are approximated by $N \times N$ matrices (YM gauge fields), which represent collectively $N^{2}$ number of points in the target space. The large $N$-limit to reproduce the continuous surface of the membrane, should be such that all the positions of the $S U(N)$ matrices are filled up in a continuous way and this limit has not been expressed, up to now, in a mathematically consistent way [16]. The non-commutative geometry of the discrete membrane is generated by the finite and discrete Heisenberg group and the space of functions on the surface of the membrane is the algebra of $N \times N$ complex matrices.

In modern language [17] the $S U(N)$ YM classical mechanics is the YM theory on noncommutative 2-torus. It is interesting that the torus compactified Matrix model is equivalent with the M-theory compactification in a constant antisymmetric background gauge field. In this case, the Matrix model description becomes that of a gauge theory on a non-commutative torus [17, 18, 19, 20, 21].

It is well known that the usual Quantum Mechanics can be represented on functions of the phase-space variables, with the Moyal bracket 22 ${ }^{1}$ replacing the classical Poisson bracket. Recently the vertex operators of open strings in an external antisymmetric gauge field $B_{\mu \nu}$ were found to obey non-commutative relations of the Weyl type, which induces a Moyal bracket structure on the space of functions on the string momenta 25].

\section{The Heisenberg-Weyl group and the Moyal bracket}

To start with, we introduce the irreducible representations of the finite Heisenberg group appropriate for the Matrix model non-commutative geometry of a toroidal membrane. The Hilbert space $\mathbb{H}_{\Gamma}$ of the wave functions on the torus $\Gamma=\mathbb{C} / \mathbb{L}$ of complex modulus $\tau=\tau_{1}+\imath \tau_{2}$, where $\mathbb{L}$ is the integer lattice, $\mathbb{L}=\left\{m_{1}+\tau m_{2} \mid\left(m_{1}, m_{2}\right) \in \mathbb{Z} \times \mathbb{Z}\right\}$ is defined as the space of functions of complex argument $z=x+\imath y$ :

$$
f(z)=\sum_{n \in \mathbb{Z}} c_{n} \mathrm{e}^{\imath \pi n^{2} \tau+2 \pi \imath n z}
$$

with norm

$$
\|f\|^{2}=\int \mathrm{e}^{-2 \pi y^{2} / \tau_{2}}|f(z)|^{2} d x d y, \quad \tau_{2}>0 .
$$

Consider the subspace $\mathbb{H}_{N}(\Gamma)$ of $\mathbb{H}_{\Gamma}$ with periodic Fourier coefficients $\left\{c_{n}\right\}_{n \in \mathbb{Z}}$ of period $N$ :

$$
c_{n}=c_{n+N} \quad n \in \mathbb{Z}, N \in \mathbb{N} .
$$

The space $\mathbb{H}_{N}(\Gamma)$ is $N$-dimensional and there is a discrete Heisenberg group, with generators $\mathcal{S}_{1 / N}$ and $\mathcal{T}_{1}$ acting as [27, 28]

$$
\left(\mathcal{S}_{1 / N} f\right)(z)=\sum_{n \in \mathbb{Z}} c_{n} \mathrm{e}^{2 \pi \imath n / N} \mathrm{e}^{2 \pi \imath n z+\pi \imath n^{2} \tau}
$$

\footnotetext{
${ }^{1}$ For a recent discussion see 23,24$]$ and references therein.

${ }^{2}$ For recent discussions see 26 .
} 


$$
\left(\mathcal{T}_{1} f\right)(z)=\sum_{n \in \mathbb{Z}} c_{n-1} \mathrm{e}^{2 \pi \imath n z+\pi \imath n^{2} \tau}, \quad c_{n} \in \mathbb{C} .
$$

On the $N$-dimensional subspace of vectors $\left(c_{1}, \ldots, c_{N}\right)$ the two generators are represented by $N \times N$ matrices, $Q, P$

$$
\left(\mathcal{S}_{1 / N}\right)_{n_{1}, n_{2}}=Q_{n_{1}, n_{2}}=\omega^{\left(n_{1}-1\right)} \delta_{n_{1}, n_{2}}, \quad\left(\mathcal{T}_{1}\right)_{n_{1}, n_{2}}=P_{n_{1}, n_{2}}=\delta_{n_{1}-1, n_{2}},
$$

with $\omega=\exp (2 \pi \imath / N)$. They satisfy the Weyl relation $Q P=\omega P Q$.

The Heisenberg group elements are defined as

$$
\mathcal{J}_{r, s}=\omega^{r \cdot s / 2} P^{r} Q^{s} .
$$

These $N \times N$ matrices are unitary $\mathcal{J}_{r, s}^{\dagger}=\mathcal{J}_{-r,-s}$ and periodic with period $N$, i.e. $\mathcal{J}_{r, s}^{N}=1$. They realize a projective representation of the discrete translation group $Z_{N} \times Z_{N}$ :

$$
\mathcal{J}_{r, s} \mathcal{J}_{r^{\prime}, s^{\prime}}=\omega^{\left(r^{\prime} s-r s^{\prime}\right) / 2} \mathcal{J}_{r+r^{\prime}, s+s^{\prime}}
$$

In ref[5] the finite $N$-Matrix model is considered as a non-commutative QM system (see also [15]), but the canonical commutation relations were not represented through the finite Heisenberg group basis $\mathcal{J}_{r, s}$. It is possible to define finite dimensional matrices $\hat{p}, \hat{q}$ such that $Q=e^{\hat{\imath}}$ and $P=e^{-\imath \hat{p}}$

$$
\hat{q}_{i j}=\frac{2 \pi}{N}(s+1-i) \delta_{i j}, \quad \hat{p}_{i j}=-\imath \frac{\pi}{N} \frac{(-1)^{(i-j)}}{\sin \frac{\pi}{N}(i-j)}
$$

where $N=2 s+1$ and $s$ is an integer. Here we have shifted by $s$ rows and columns of $Q$ and $P$ matrices defined in relations (5). These matrices satisfy new Heisenberg commutation relations, which have a very simple form 29]

$$
-\imath[\hat{q}, \hat{p}]_{i j}=\frac{2 \pi}{N} \frac{\frac{\pi}{N}(i-j)(-1)^{(i-j)}}{\sin \frac{\pi}{N}(i-j)}
$$

when $i \neq j$ and zero when $i=j$. The matrix $\hat{q}$ satisfies the torus compactification relations of the Matrix model, with corrections due to their finite size

$$
P^{-1} \hat{q} P=\hat{q}+\frac{2 \pi}{N} I_{N}-2 \pi I_{0}
$$

where $I_{N}$ is the $N \times N$ identity matrix and $I_{0}$ the $N \times N$ diagonal matrix with diagonal elements $\{1,0, \ldots, 0\}$.

The bosonic part of the matrix model is the $S U(N)$ YM classical mechanics and the gauge fields are linear combinations of the elements $\mathcal{J}_{r, s}$, i.e.,

$$
A_{l}(t)=\sum_{r, s=0}^{N-1} A_{l}^{r, s} J_{r, s}, \quad l=1, \ldots, d-1
$$


which can be considered as coherent states of the discrete and finite toroidal phase-space $N \times N$ lattice. The $A_{l}$ matrices are the non-commutative coordinates of the discrete membrane in $d-1$ dimensions.

There is another representation of the standard quantum mechanics on the space of functions of the phase-space variables. This is the unique deformation of the Poisson bracket, the Moyal bracket 22, 24]

$$
\{\{f, g\}\}_{\lambda}(u, v)=\left.\frac{1}{\lambda} \sin \left(\lambda\left(\partial_{u} \partial_{v^{\prime}}-\partial_{u^{\prime}} \partial_{v}\right)\right) f(u, v) g\left(u^{\prime}, v^{\prime}\right)\right|_{u=u^{\prime}, v=v^{\prime}}
$$

Here, $\lambda$ corresponds to the Planck constant and the Moyal bracket gives a structure of infinite dimensional algebra on the space of functions on the torus generated by

$$
e_{r, s}(u, v)=\frac{1}{2 \pi} e^{\imath(r u+s v)}
$$

where $u, v \in[0,2 \pi]$ and $r, s \in \mathbb{Z}$. This algebra is the trigonometric algebra of Fairlie Fletcher and Zachos 30]:

$$
\left\{\left\{e_{r, s}, e_{r^{\prime}, s^{\prime}}\right\}\right\}_{\lambda}(u, v)=\frac{1}{2 \pi \lambda} \sin \left(\lambda\left(r s^{\prime}-r^{\prime} s\right)\right) e_{r+r^{\prime}, s+s^{\prime}}(u, v)
$$

which also icludes the case $\lambda=\frac{2 \pi}{N}$. This case gives the $S U(N)$ algebra in the base $\mathcal{J}_{r, s}$ :

$$
\left[\mathcal{J}_{r, s}, \mathcal{J}_{r^{\prime}, s^{\prime}}\right]=-2 \imath \sin \left(\frac{2 \pi}{N}\left(r s^{\prime}-r^{\prime} s\right)\right) \mathcal{J}_{r+r^{\prime}, s+s^{\prime}}
$$

if the $e_{r, s}$ functions are identified with $e_{r+k N, s+m N}$ for $k, m \in \mathbb{Z}$. The Heisenberg group matrices $\mathcal{J}_{r, s}$ have been introduced by Weyl.

When $\lambda \rightarrow 0$ (or $N \rightarrow \infty$ ), we recover the Poisson algebra of the area preserving transformations of the torus [31]

$$
\left\{e_{r, s}, e_{r^{\prime}, s^{\prime}}\right\}(u, v)=\left(r^{\prime} s-r s^{\prime}\right) \frac{1}{2 \pi} e_{r+r^{\prime}, s+s^{\prime}}
$$

The Matrix model has various large $N$-limits. Up to now it is not known how to get the quantum mechanics of supermembrane starting from this model, even though, various compactifications indicate that it has membrane states as excitations. We believe that the appropriate limit is the 't Hooft topological expansion of the $S U(N)$ YM-mechanics. To this end, we shall determine what happens to the Heisenberg group matrices $\mathcal{J}_{r, s}$ in this limit. We observe that these matrices contain powers of the root of unity along two diagonals so we start with $\omega=e^{2 \pi \imath \frac{M}{N}},(M, N$ co-prime integers $)$. The correct large $N$-limit for $S U(N)$ is the inductive one, i.e., $S U(N) \rightarrow S U(N+1) \rightarrow S U(N+2) \ldots$ which we get if we let $M, N \rightarrow \infty$ with $M / N=$ constant. Note that the constant $\hbar=2 \pi \frac{M}{N}$ can be identified with the flux of the 3 -index antisymmetric gauge field per unit membrane area. The Weyl relations become the Heisenberg group relations for an infinite phase-space lattice and in the Fourier transform space of both canonical variables the Matrix model 
describes a toroidal continuous membrane with Matrix commutators replaced by Moyal brackets [32]. Since the limit $\hbar \rightarrow 0$ replaces the Moyal bracket by Poisson, we get from Moyal YM theory the membrane. Higher order corrections to $\hbar$ can be represented as membranes with attached handles on the initial membrane which is determined by the $S U(N)$ chosen basis, in our case the torus.

In this limit, the light-cone gauge equations of motion for the membrane

$$
\ddot{X}_{i}=\left\{X_{k},\left\{X_{k}, X_{i}\right\}\right\} ; i, k=1, \ldots, d-1
$$

and the corresponding Gauss law $\left\{X_{i}, \dot{X}_{i}\right\}=0$ are replaced by

$$
\begin{aligned}
\ddot{X}_{i} & =\left\{\left\{X_{k},\left\{\left\{X_{k}, X_{i}\right\}\right\}\right\}\right\} \\
\left\{\left\{X_{i}, \dot{X}_{i}\right\}\right\} & =0, i, k=1, \ldots d-1 .
\end{aligned}
$$

When the space of functions on the toroidal membrane is replaced by the algebra of $N \times N$ matrices, the coordinates of the membrane become the matrices $A_{i}(t)$, the velocity is the $S U(N)$ electric field $E_{i}(t)=\dot{A}_{i}(t)$, and the magnetic field in three or seven dimensions is

$B_{i}(t)=\frac{1}{2} f_{i j k}\left[A_{j}, A_{k}\right]$ where $f_{i j k}$ is the $\epsilon_{i j k}$ totally antisymmetric symbol in three dimensions and $\Psi_{i j k}$ the octonionic multiplication table in seven dimensions [33].

The Moyal bracket generalizes both Poisson brackets and matrix commutators, so that one is tempted to consider a system where the Poisson bracket is replaced by the Moyal one [34]. The question of the appearance of Moyal bracket for physical reasons in the dynamics of membrane is up to now open. We know that there are other limits of the Matrix model, one leads to perturbative string field theory [35, 36], and the Poisson limit in which the $S U(N)$ symmetry becomes the area-preserving diffeomorphism group. We believe that the physical origin of the Moyal bracket is due to the presence of the antisymmetric background field $C_{i j k}$ in the light-cone gauge which gives a 'magnetic' flux (Hall effect), trasforming the surface of the membrane into a non-commutative phase-space[37]. This is true for open membranes where the topological term of the action receives a contribution from the boundary.

\section{Topological charge, Bogomol'nyi bound and Integrability.}

In order to explain the appearance of non-abelian electric-magnetic type of duality in the membrane theory, we recall that for YM-potentials independent of space coordinates the self-duality equation in the gauge $A_{0}=0$ is

$$
\dot{A}_{i}=\frac{1}{2} \epsilon_{i j k}\left[A_{j}, A_{k}\right], i, j, k=1,2,3
$$

According to ref 38 the only non-trivial higher dimensional YM self-duality equations exist in 8 space-time dimensions which, for the 7-space coordinate independent potentials, can be written (in the $A_{0}=0$ gauge) as

$$
\dot{A}_{i}=\frac{1}{2} \Psi_{i j k}\left[A_{j}, A_{k}\right], i, j, k=1, \cdots 7
$$


where $\Psi_{i j k}$ is the multiplication table of the seven imaginary octonionic units.

It is now tempting to take the large $N$-limit and replace the commutators by Poisson (Moyal) brackets to obtain the self-duality equations for membranes (non-commutative instantons for the Moyal case). In this limit we replace the gauge potentials $A_{i}$ by the membrane coordinates $X_{i}$. Then, the 3 -d system is 39, 40],

$$
\dot{X}_{i}=\frac{1}{2} \epsilon_{i j k}\left\{X_{j}, X_{k}\right\}, i, j, k=1,2,3,
$$

while in seven space dimensions 34, 41]

$$
\dot{X}_{i}=\frac{1}{2} \Psi_{i j k}\left\{X_{j}, X_{k}\right\}, i, j, k=1, \cdots, 7
$$

and correspondingly for the case of Moyal brackets in three dimensions 42, 43 and in seven dimensions [24]. It is easy to see that the self-duality membrane equations, imply the second order Euclidean-time, equations of motion in the light-cone gauge as well as the Gauss law.

One striking feature of the self-duality membrane equations is their simple geometrical meaning 39, 41]. These equations state that the normal vector at a point of the membrane surface and the velocity at the same point are parallel (self-dual) or anti-parallel (anti-selfdual). The possibility to write down self-duality equations based on the existence of vector cross-product comes from the existence of the quaternionic and octonionic algebras. Since these are the only existing division algebras the 3 and 7 dimensions are unique 33 . The validity of this geometrical statement could be extended in a general curved space-time background as a definition of the self-dual membranes.

If one takes the limit where the commutator of matrices is replaced by commutator of operators or the Moyal bracket, then the self-duality equations become the Moyal Nahm or Moyal-Bogomol'nyi equations of [34].

The membrane instantons carry a topological charge density [45] which satisfies a Bogomol'nyi bound [46]:

$$
\Omega(X)=\frac{1}{3 !} \epsilon^{a b c} f_{i j k} X_{a}^{i} X_{b}^{j} X_{c}^{k}
$$

where $X_{a}^{i}=\partial_{\xi_{a}} X^{i}, a, b, c=1,2,3$ and $f_{i j k}=\epsilon_{i j k}$ when $i, j, k=1,2,3$ and $f_{i j k}=\Psi_{i j k}$ for $i, j, k=1, \cdots, 7$. This topological charge density defines the topological charge of the membrane

$$
Q=\frac{1}{V_{3}} \int d^{3} \xi \Omega(X)
$$

where $V_{3}$ is the volume of the integration region. The topological charge $Q$ is an integer and represents the degree of the map from the membrane to its world volume. We display below the convenient representation of the topological charge which will help us demonstrate that it is a lower bound of the membrane action for topologically non-trivial membranes

$$
\Omega(X)=\frac{1}{2} \dot{X}_{i} f_{i j k}\left\{X^{j}, X^{k}\right\}=\frac{1}{2}\left\{X^{j}, X^{k}\right\}^{2}
$$

\footnotetext{
${ }^{3}$ For other approach to self-duality see also 44 .
} 
where the self-duality equations as well as the properties of $f_{i j k}$ in three and seven dimensions have been used. The topological charge of the membrane can now be written as

$$
Q=\frac{1}{2 V_{3}} \int_{M} d^{3} \xi\left\{X^{j}, X^{k}\right\}^{2}
$$

The minimum value of $Q(Q=1)$ is obtained for the membrane instanton compactified on a world-volume torus, $X_{1}=\sqrt{2} \sigma_{1}, X_{2}=\sqrt{2} \sigma_{2}$ and $X_{3}=2 t$.

The Euclidean action can be written as

$$
S=\frac{1}{V_{3}} \int d^{3} \xi\left(\frac{1}{2} \dot{X}_{i}^{2}+\frac{1}{4}\left\{X_{j}, X_{k}\right\}^{2}\right)
$$

From the inequality $\left(\dot{X}_{i} \pm \frac{1}{2} f_{i j k}\left\{X_{j}, X_{k}\right\}\right)^{2} \geq 0$ we derive that,

$$
S \geq Q
$$

and the equality holds only for the self-dual or anti-self-dual membranes. So the self-dual or anti-self-dual membranes are BPS Euclidean-time membrane world-volume solitons. As we have seen in ref 47, the $3-d$ and 7 -d self-dual solutions preserve 8 and 1 supersymmetries respectively or $1 / 2$ and $1 / 16^{\text {th }}$ of the supersymmetry of the light-cone supermembrane Hamiltonian. This is a direct consequence of the above Bogomol'nyi bound and the $S O(3)$ and $G_{2}$ rotational space symmetry of the above cases.

The role of the membrane instantons is important in developing a perturbative expansion. Configurations of the membrane around instantons cannot collapse to points or strings, because they have different topological charge. The 3 -index antisymmetric gauge field which is so crucial for the uniqueness of the supermembrane Lagrangian participates in the bosonic part through the Cern-Simons term. If its vacuum expectation value is non-zro and proportional to $\Psi_{i j k}$ (in the corresponding 7 dimensions), then the topological charge defined above, separates the functional integral into membrane topological sectors. Going now to the case of Moyal-Nahm equations, there is a corresponding topological charge without an obvious geometrical meaning and the Bogomol'nyi bound is valid in this case too. This bound is important for the stability of the corresponding Moyal-Nahm instantons. Recent discussions on the role of instantons in non-commutative YM theories (non-commutative instantons) imply that they can be considered as regularizations of small size instantons in standard YM theories (see e.g. [48]). The case of Moyal-Nahm equations could be considered as non-commutative membrane instantons which regularize the Poisson or membrane case.

We now make few remarks on the integrability of the self-dual equations. The 3-d selfduality system has a Lax pair and an infinite number of conservation laws [39, 40]. In order to see this, we first rewrite the self-duality equations in the form

$$
\dot{X}_{+}=i\left\{X_{3}, X_{+}\right\}, \quad \dot{X}_{-}=i\left\{X_{3}, X_{-}\right\}, \quad \dot{X}_{3}=\frac{1}{2} i\left\{X_{+}, X_{-}\right\}
$$


where

$$
X_{ \pm}=X_{1} \pm i X_{2}
$$

The Lax pair equations can be written as

$$
\dot{\psi}=L_{X_{3}+\lambda X_{-}} \psi, \quad \dot{\psi}=L_{\frac{1}{\lambda} X_{+}-X_{3}} \psi
$$

where the differential operators $L_{f}$ are defined as

$$
L_{f} \equiv i\left(\frac{\partial f}{\partial \phi} \frac{\partial}{\partial \cos \theta}-\frac{\partial f}{\partial \cos \theta} \frac{\partial}{\partial \phi}\right)
$$

The compatibility condition of (32) is

$$
\left[\partial_{t}-L_{X_{3}+\lambda X_{-}}, \partial_{t}-L_{\frac{1}{\lambda} X_{+}-X_{3}}\right]=0
$$

from which, comparing the two sides for the coefficients of the powers $\frac{1}{\lambda}, \lambda^{0}, \lambda^{1}$ of the spectral parameter $\lambda$, we find (30). From the linear system (32), using the inverse-scattering method, one could in principle construct all solutions of the self-duality equations.

The infinite number of conservation laws are derived as follows: from the Cartesian formulation

$$
\frac{d X_{i}}{d t}=\frac{1}{2} \epsilon_{i j k}\left\{X_{j}, X_{k}\right\}
$$

contracting with a complex 3 -vector $u_{i}$ such that

$$
u_{i}=\epsilon_{i j k} u_{j} v_{k}
$$

where $u_{i} u_{i}=0$, and $v$ is another complex vector with $v_{i} v_{i}=-1$ and $u_{i} v_{i}=0$, we find,

$$
\frac{d u \cdot X}{d t}=\{u \cdot X, v \cdot X\}
$$

The latter is a Lax pair type equation, which implies

$$
\frac{d}{d t} \int d^{2} \xi(u \cdot X)^{n}=0
$$

Applying the same method in seven dimensions with two complex 7-vectors $u_{i}, v_{i}$ such that $u_{i} u_{i}=0, v_{i} v_{i}=-1$ and $u_{i} v_{i}=0$, leads to the equation

$$
\frac{d u \cdot X}{d t}=\{u \cdot X, v \cdot X\}+\frac{1}{2} \phi_{j k l m} u_{j} v_{k}\left\{X_{l}, X_{m}\right\}
$$

The curvature tensor $\phi_{j k l m}$ is defined as the dual of $\Psi_{i j k}$ in seven dimensions. When equation (39) is restricted to three dimensions we recover (37). We observe that the presence of the curvature tensor is an obstacle for the integrability. At this point, we may look for an extended definition of integrability replacing the zero-curvature condition with the 
octonionic curvature one. We can restrict the above equation in particular subspaces of solutions where integrability appears. One possibility is the factorization of the time [41].

We conclude with a few remarks. In this note we have given arguments that the Matrix model describes a non-commutative YM theory for the supermembrane in the presence of background three-index antisymmetric gauge fields. If this conjecture is true, it implies that the excitations of this model in various compactifications are also physical excitations of the supermembrane. So the supermembrane should contain 11-d supergravity at least in weak coupling limits given by small radii of the compactification manifolds. It is tempting to calculate correlation functions of membrane observables using the Matrix model and then take the large $N$-limit as was discussed in section 3. On the other hand, perturbation theory for the supermembrane could be defined through the expansion in the parameter $\hbar / N$, with $M / N \rightarrow \hbar$ for $M, N \rightarrow \infty$. In this expansion all the topologies of the membrane appear as splitting and joining interactions The other known large $N$-limit [35, 36] gives the string perturbation theory as a QM sector of the supermembrane.

As this work was written, we have been kindly informed that the Moyal limit of the Matrix model has been studied in connection with the higher derivative corrections to the Born-Infeld Lagrangians for the D2-brane 49. For a very recent, interesting paper on D-branes in group manifolds, see 50

One of us (EGF) would like to thank prof. Albert Schwarz for a valuable discussion.

\section{References}

[1] E. Witten, Nucl. Phys. B460 (1996) 335 hep-th/9510135.

[2] P.K. Townsend, Phys. Lett. B373 (1996) 68 hep-th/9512062.

[3] E. Bergshoeff, E. Sezgin and P.K. Townsend, Phys. Lett. B189 (1987) 75.

[4] B. de Wit, hep-th/9902051.

[5] T. Banks, W. Fischler, S.H. Shenker and L. Susskind, Phys. Rev. D55 (1997) 5112 hep-th/9610043.

[6] N. Ishibashi, H. Kawai, Y. Kitazawa and A. Tsuchiya, Nucl. Phys. B498 (1997) 467 hep-th/9612115.

[7] A.P. Polychronakos, Phys. Lett. B403 (1997) 239 hep-th/9703073.

[8] G. Goldstone, MIT-1980, Unpublished.

[9] J. Hoppe, MIT-Ph. D. Thesis, (1982) Elem. Part. Research Journal Kyoto, 83 $(1989 / 90) 3$. 
[10] S.G. Matinian, G.K. Savvidy and N.G. Ter-Arutunian Savvidy, Sov. Phys. JETP 53 (1981) 421.

[11] B. Simon, Annals Phys. 146 (N.y.) 209.

[12] E. Bergshoeff, E. Sezgin, Y. Tanii and P.K. Townsend, Ann. Phys. 199 (1990) 340.

[13] B. de Wit, J. Hoppe and H. Nicolai, Nucl. Phys. B305 (1988) 545.

[14] B. de Wit, M. Luscher and H. Nicolai, Nucl. Phys. B320 (1989) 135.

[15] E.G. Floratos, Phys. Lett. B228 (1989) 335.

[16] E.G. Floratos and J. Iliopoulos, to appear.

[17] A. Connes, M.R. Douglas and A. Schwarz, JHEP 02 (1998) 003 hep-th/9711162.

[18] C. Hofman and E. Verlinde, Nucl. Phys. B547 (1999) 157 hep-th/9810219.

[19] M.R. Douglas, hep-th/9901146.

[20] M.R. Douglas and C. Hull, JHEP 02 (1998) 008 hep-th/9711165.

[21] P. Ho and Y. Wu, Phys. Rev. D58 (1998) 066003 hep-th/9801147.

[22] J.E. Moyal, Proc. Cambridge Phil. Soc. 45 (1949) 99.

[23] C. Zachos and T. Curtright, hep-th/9903254.

[24] D.B. Fairlie, Mod. Phys. Lett. A13 (1998) 263 hep-th/9707190.

[25] V. Schomerus, JHEP 06 (1999) 030 hep-th/9903205.

[26] E. Witten, N. Seiberg, Talks in Strings 99, Potsdam July 1999, http://string99.aei-potsdam.mpg.de

[27] P. Cartier, "Quantum Mechanical Commutation Relations and Theta Functions" in Proc. Symp. Pure Mathematics, 9: Algebraic-Discontinuous Groups, AMS, Providence, RI (1966).

[28] D. Mumford, Tata Lectures on Theta, I-III, Birkhäuser, New York (1986).

[29] E.G. Floratos and G.K. Leontaris, Phys. Lett. B412 (1997) 35 hep-th/9706156.

[30] D.B. Fairlie, P. Fletcher and C.K. Zachos, Phys. Lett. B218 (1989) 203.

[31] E.G. Floratos, J. Iliopoulos and G. Tiktopoulos, Phys. Lett. B217 (1989) 285.

[32] S.R. Das, A. Dhar, G. Mandal and S.R. Wadia, Mod. Phys. Lett. A7 (1992) 71 hepth/9111021.

A. Kavalov and B. Sakita, Annals Phys. 255 (1997) 1 hep-th/9603024. 
[33] R. Dundarer, F. Gursey and C. Tze, J. Math. Phys. 25 (1984) 1496.

[34] T. Curtright, D. Fairlie and C. Zachos, Phys. Lett. B405 (1997) 37 hep-th/9704037.

[35] R. Dijkgraaf, H. Verlinde and E. Verlinde, Nucl. Phys. Proc. Suppl. 68 (1998) 28.

[36] G. Bonelli, L. Bonora, F. Nesti and A. Tomasiello, hep-th/9905092.

[37] G. Chapline and A. Granik, hep-th/9808033.

[38] E. Corrigan, C. Devchand, D.B. Fairlie and J. Nuyts, Nucl. Phys. B214 (1983) 452.

[39] E.G. Floratos and G.K. Leontaris, Phys. Lett. B223 (1989) 153.

[40] R.S. Ward, Phys. Lett. B234 (1990) 81.

[41] E.G. Floratos and G.K. Leontaris, Nucl. Phys. B512 (1998) 445 hep-th/9710064.

[42] C. Castro and J. Plebanski, J. Math. Phys. 40 (1999) 3738 hep-th/9710041.

[43] H. Garcia-Compean and J.F. Plebanski, Phys. Lett. A234 (1997) 5 hep-th/9612221.

[44] T.A. Ivanova and A.D. Popov, Theor. Math. Phys. 102 (1995) 280.

[45] B. Biran, E.G. Floratos and G.K. Savvidy, Phys. Lett. 198B (1987) 329.

[46] C. Zachos, D. Fairlie and T. Curtright, hep-th/9709042.

[47] E.G. Floratos and G.K. Leontaris, Phys. Lett. B428 (1998) 75 hep-th/9802018.

[48] N. Nekrasov and A. Schwarz, Commun. Math. Phys. 198 (1998) 689 hep-th/9802068.

[49] L. Cornalba and R. Schiappa, hep-th/9907211.

[50] H. Garcia-Compean and J.F. Plebanski, hep-th/9907183. 